\title{
An Integrated Approach to Grouping and Matching
}

\author{
Richard C Wilson and Edwin R Hancock \\ Department of Computer Science, \\ University of York, York Y01 5DD,UK.
}

\begin{abstract}
Perceptual grouping and relational matching are conventionally viewed as sequential stages of effective intermediate level scene interpretation. Relational structures established on the basis of perceptual grouping criteria are utilised in a bottom-up control strategy and hence form the input data representation for subsequent matching. Our standpoint in this paper is that the two processes should be tightly coupled to one-another so that the relational model can prevail upon the extraction of perceptual groupings, providing additional constraints on an otherwise potentially fragile processing operation. We realise this objective by casting the grouping and matching operations as an iterative discrete relaxation process. The dual operations of re-organising the perceptual relation graph and subsequent matching against a model, both optimise a single objective function in the maximum a posteriori probability sense. Grouping and matching are therefore cast into an integrated optimisation framework that is realised by tightly coupled update processes.
\end{abstract}

\section{Introduction}

Perceptual grouping [4] and relational matching $[1,3,5]$ are processes of central importance in the established hierarchy of computer vision operations. Conventionally perceptual grouping is used to establish relational data structures for later matching. Viewed from this perspective grouping is a process of structural filtering aimed at removing relational clutter. When applied effectively, the grouping process may significantly enhance the fidelity of subsequent matching operations [4].

Although component parts of a clearly established hierarchy, grouping and matching are invariably viewed in a bottom-up perspective [4]. Little interaction or feedback exists other than by virtue of the coupling induced by the vertical propagation of a common data abstraction. Moreover, this abstraction is usually devoid of any evidential index associated with the significance of perceptual tokens, Our viewpoint in this paper is that there are important symbiotic advantages to be gained from the tight integration and feedback between the two processes. We aim to demonstrate that relational constraints provided by a model can prevail upon the effective extraction of meaningful perceptual groupings from otherwise heavily corrupted and distorted data through the propagation of an index of evidential significance. By deleting redundant perceptual tokens we aim to reconstruct corrupted relations and hence improve the quality of final match. 
Our framework for realising this objective of an integrated approach to grouping and matching is provided by maximum a posteriori probability (MAP) estimation [2]. We assess the effects of both modifying the set of perceptual tokens and updating their matched realisation using a Bayesian measure of evidential significance [5]. Simultaneous optimisation of relational structure and consistency of match is realised by two distinct classes of update operations. The first of these involves deleting and reinstating nodes from the relational graphs representing the salient perceptual structures [4]. This structural grouping process is one of dynamic graph reconfiguration which takes place by direct reference to the raw image data. Once a graph node has been identified as being relationally redundant, the corresponding perceptual token is deleted from the data and the grouping is recomputed. The second class of updating operations maintain a consistent match between the prevailing grouping of perceptual entities in the data and the available relational model. In this way the processes of perceptual grouping and matching interact through a common objective function. Effectively, the dual update processes are integrated together in a unified optimisation framework.

The outline of this paper is follows. Section 2 introduces the formal ingredients and computational framework underlying our method. We present an experimental evaluation of our method in Section 3. Finally, Section 4 offers some conclusions.

\section{Computational Framework}

We abstract the matching of perceptual structures in terms of attributed relational graphs [1]. According to this representation the nodes are entities to be matched. The arcs represent perceptual relations operating between the nodes. We use the triple $G=(V, E, \mathcal{A})$ to denote the graphs under match, where $\mathrm{V}$ is the set of nodes, $E \subset V \times V$ is the set of arcs and $\mathcal{A}=\left\{\mathbf{x}_{i}, \forall i \in V\right\}$ is a set of unary measurements associated with the nodes. Our aim in matching is locate correspondences between the nodes in a graph $G_{1}=\left(V_{1}, E_{1}, \mathcal{A}_{1}\right)$ representing the perceptual organisation of the data and those in a graph $G_{2}=\left(V_{2}, E_{2}, \mathcal{A}_{2}\right)$ representing the available relational model. Formally, the matching is represented by a function $f: V_{1} \rightarrow V_{2}$ from the nodes in the data graph $G_{1}$ to those in the model graph $G_{2}$.

In performing the matches of the nodes in the data graph $G_{1}$ we will be interested in exploiting structural constraints provided by the model graph $G_{2}$. In order to strike a compromise between exploiting constraints of maximum power, on the one hand, and initiating an uncontrolled explosion of computational complexity, on the other, we will use subgraphs that consist of neighbourhoods of nodes interconnected by arcs; for convenience we refer to these structural subunits or $\mathrm{N}$-ary relations as supercliques. The superclique of the node indexed $j$ in the graph $G_{1}$ with arc-set $E_{1}$ is denoted by the set of nodes $C_{j}=j \cup\left\{i \mid(i, j) \in E_{1}\right\}$. We use the notation $R_{j}=\left(u_{1}, u_{2}, \ldots, u_{\left|C_{j}\right|}\right)$ to denote the $\mathrm{N}$-ary symbolic relation represented by the nodes of the superclique $C_{j} \subset V_{1}$ 
in the data graph $G_{1}$. The matched realisation of this superclique is denoted by the relation $\Gamma_{j}=\left(f\left(u_{1}\right), f\left(u_{2}\right), \ldots, f\left(u_{\mid C_{j}} \mid\right)\right)$. The critical ingredient in developing our matching scheme is the set of structure preserving mappings between each superclique of graph $G_{1}$ and those of graph $G_{2}$. The set of mappings, or dictionary, for the superclique $C_{j}$ is denoted by $\Theta\left(C_{j}\right)=\left\{S_{i}\right\}$ where each element $S_{i}$ of $\Theta\left(C_{j}\right)$, is a relation formed on the nodes of the model graph. We denote such consistent symbolic relations by $S_{i}=\left(v_{1}, v_{2}, \ldots\right)$.

Our aim is to cast the processes of perceptual organisation and relational matching into a unified optimisation framework. This combined optimisation process involves two classes of update operation. The first of these is the iterative reconstruction of the relational graph $G_{1}$ by node deletions and reinsertions to improve the overall grouping of perceptual entities. The second class of update operations are concerned with modifying the match so as to maintain consistency with the relational model $G_{2}$.

At the computational level, these dual update operations involve maintaining two separate representations of the perceptual relation graph. Suppose that $G_{1}=$ $\left(E_{1}, V_{1}, \mathcal{A}_{1}\right)$ is the current representation of the perceptual organisation of the data. Graph $G_{1}^{\prime}=\left(E_{1}^{\prime}, V_{1}^{\prime}, \mathcal{A}_{1}^{\prime}\right)$, on the other hand, is the reconfigured relational structure formed by excluding the single perceptual entity represented by node $u$ in $G_{1}$ and reassigning it to the null or outlier set $\Phi$. The node-set $V_{1}^{\prime}=V_{1}-u$ and the attribute-set $\mathcal{A}_{1}^{\prime}=\mathcal{A}_{1}-\mathbf{x}_{u}^{1}$ are trivially recomputed by deleting the entry associated with node $u$. Determination of the edge-set $E_{1}^{\prime}$ is more complex and must be undertaken by recomputing the relational affinity of the nodes when the perceptual entity associated with node $u$ is excluded from the raw image data. The match between the reconfigured graph $G_{1}^{\prime}$ and the model graph $G_{2}$ is represented by the function $f^{\prime}: V_{1}^{\prime} \rightarrow V_{2}$.

\subsection{MAP Criterion}

From the standpoint of information theory, we therefore seek the grouped and matched configuration of nodes that has maximum a posteriori probability with respect to the available unary measurement information. Computationally, the objective is to formulate iterative local decision schemes that are capable of both grouping and matching nodes. More formally, these iterative reassignment processes are aimed at partitioning the set of entities into those that are grouped to form a consistently matched graph $f$ and and those that are assigned to a set of relational outliers $\Phi$. In other words, we seek the grouped and matched configuration of nodes that optimise the quantity $P\left(f, \Phi \mid \mathcal{A}_{1}, \mathcal{A}_{2}\right)$. Our development of a detailed MAP update process proceeds from the starting point described in [2] and centres around dual assumptions concerning the matching process. Firstly, we assume conditional independence over the unary measurements. Of greater significance to our unified grouping and matching process is the assumption that we can factorise the joint prior $P(f, \Phi)$ over the set of matched nodes and the set of outliers, i.e. $P(f, \Phi)=P(f) \cdot P(\Phi)$.

In order to realise the dual processes of grouping and matching, we require a model of the joint prior $P(f)$. In the context of relational matching, we have 
recently described a Bayesian model of the joint prior which draws on the structure preserving relational mappings contained within the dictionary [5]. Central to this process is the idea of gauging relational consistency using the Hamming distance between an assigned match $\Gamma_{j}$ and a structure preserving mapping $S_{i}$ by the Hamming distance $H\left(\Gamma_{j}, S_{i}\right)=\sum_{i \in C_{j}}\left(1-\delta_{f\left(u_{i}\right), v_{i}}\right)$. With this ingredient, the joint prior for the matching configuration $\Gamma_{j}$ is equal to

$$
P\left(\Gamma_{j}\right)=\frac{K_{C_{j}}}{\left|\Theta\left(C_{j}\right)\right|} \sum_{S_{i} \in \Theta\left(C_{j}\right)} \exp \left[-k_{e} H\left(\Gamma_{j}, S_{i}\right)\right]
$$

where $K_{C_{j}}=\left(1-P_{e}\right)^{\left|C_{j}\right|}$ and the exponential constant is related to the probability of memoryless matching errors $P_{e}$ by $k_{e}=\ln \frac{1-P_{e}}{P_{e}}$. The joint prior $P(f)$ is computed by averaging the clique priors $P\left(\Gamma_{j}\right)$ over the nodes of the data graph. Our model of the joint prior for the outlier set is based on the assumption that is contains no meaningful relational structure and that $P(\Phi)=P_{\phi}^{|\Phi|}$, where $P_{\phi}$ is the uniform probability of relational outliers.

\subsection{Coupled Updates}

In elucidating our grouping process, we make the observation that in order to gauge the net effect of deleting a node using the consistency measure given by equation (12) we need only examine those contributions that arise from the modified superclique set. This set is constructed by identifying those nodes that form a superclique with node $u$ in graph $G_{1}$, i.e. $C_{u}-\{u\}$, and determining the new superclique set for these nodes in the reconfigured graph $G_{1}^{\prime}$. We let $\chi_{u}^{+}$denote the superclique set of object $u$ in graph $G_{1}$ and $\chi_{u}^{-}$denote the corresponding superclique set in the reconfigured graph $G_{1}^{\prime}$. With this notation the change in the MAP criterion caused by the deletion of the node $u$ is proportional to

$$
\Delta_{u}^{-}=P_{\phi} \sum_{j \in \chi_{\bar{u}}} P\left(\Gamma_{j}\right)
$$

By contrast, when considering the change in the MAP criterion due to the re-insertion of node $u$ it is the superclique set $\chi_{u}^{+}$to which we turn our attention. The quantity of interest depends not only upon the joint matching priors $P\left(\Gamma_{j}\right)$ but also upon the a posteriori matching probabilities $P\left(u, v \mid \underline{x}_{u}^{1}, \mathbf{x}_{v}^{2}\right)$ and single node priors $P(u, v)$

$$
\Delta_{u}^{+}=\frac{P\left(u, v \mid \mathbf{x}_{u}^{1}, \mathbf{x}_{v}^{2}\right)}{P(u, v)} \sum_{j \in \chi_{u}^{+}} P\left(\Gamma_{j}\right)
$$

With these two measures to hand, we can both delete and reinstate nodes in such a way as to monotonically increase the MAP criterion. We therefore delete node $u$ provided $\Delta_{u}^{+}<\Delta_{u}^{-}$and reinstate the node if $\Delta_{u}^{+}>\Delta_{u}^{-}$.

The graph reconfiguration process described above is aimed at restoring meaningful relational groupings using constraints provided by a model-graph. 
With this enhanced relational structure to hand, a more consistent match may be recovered. In commencing the grouping process, we have assumed that a match of maximum achievable consistency had already been established for the mapping $f$. A consistent match may be maintained in the MAP sense provided that the mapping $f$ is continually updated as follows

$$
f(u)=\arg \max _{v \in V_{2}} \frac{P\left(u, v \mid \mathbf{x}_{u}^{1}, \mathbf{x}_{v}^{2}\right)}{P(u, v)} \sum_{i \in \chi_{u}^{+}} P\left(\Gamma_{i}\right)
$$

Relational consistency is therefore incrementally maintained provided that we update those matches for the nodes in supercliques modified by the deletion or insertion processes.

\section{Experiments}

Evaluation of our unified matching process is concerned with matching hedge structures segmented from synthetic aperture radar (SAR) images against their cartographic representation in a digital Ordnance Survey map. The initial matches between the linear segments extracted from the SAR data and their map representation are established on the basis of the affinity between the vectors of unary node attributes in the two graphs. The matching probabilities are computed from exponential distributions of the Mahalanobis distance between attribute-vector pairs computed using an estimate of the variance-covariance matrix $\Sigma$, i.e.

$$
P\left(u, v \mid \mathbf{x}_{u}^{1}, \mathbf{x}_{v}^{2}\right)=\left(1-P_{\phi}\right) \frac{\exp \left[-\frac{1}{2}\left(\mathbf{x}_{u}^{1}-\mathbf{x}_{v}^{2}\right)^{T} \Sigma^{-1}\left(\mathbf{x}_{u}^{1}-\mathbf{x}_{v}^{2}\right)\right]}{\sum_{w \in V_{2}} \exp \left[-\frac{1}{2}\left(\mathbf{x}_{u}^{1}-\mathbf{x}_{w}^{2}\right)^{T} \Sigma^{-1}\left(\mathbf{x}_{u}^{1}-\mathbf{x}_{w}^{2}\right)\right]}
$$

Our assummed model for the matching priors is one of uniformity over the set of matches i.e. $P(u, v)=\left(\left|V_{1}\right| \cdot\left|V_{2}\right|\right)^{-1}$.

The experimental matching study is based on 95 linear segments in the SAR data and 30 segments contained in the map. However only 23 of the SAR segments have feasible matches within the map representation. Figure 1 illustrates the results of applying the unified grouping and matching technique. Figure 1a. shows the original SAR image. Figure 1b. shows the line segments extracted from the digital map. Figure $1 c$ shows the initial matches for the corresponding line segments in the SAR data; the black lines are correct matches while the grey lines are matching errors. With the same coding scheme Figure 1d. shows the final result once the iterative grouping and matching process has converged. Here those lines assigned to the set $\Phi$ have been deleted from the figure. Comparing Figures 1c. and 1d. it is clear that the main effect of simultaneous grouping and matching operations, has been to delete the majority of the clutter segments from the SAR data graph. To give some idea of relative performance merit, in the case of the initial matching configuration 20 of the 23 matchable segments are correctly identified with 75 incorrect matches, while after application of the unified grouping method the final graph contains 19 correct matches, only 17 residual clutter nodes and 59 deleted nodes. 
a)

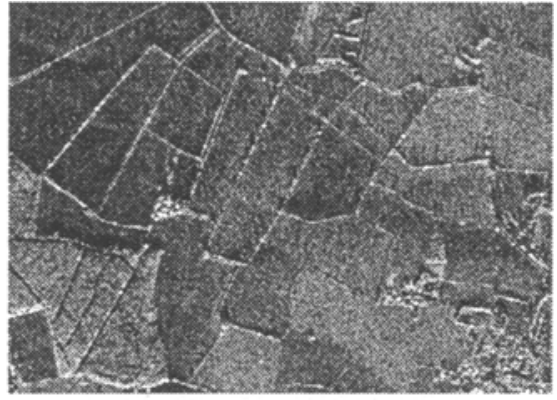

c) $\cdots$

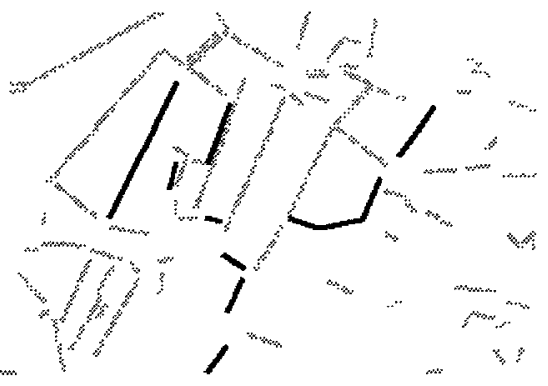

b)

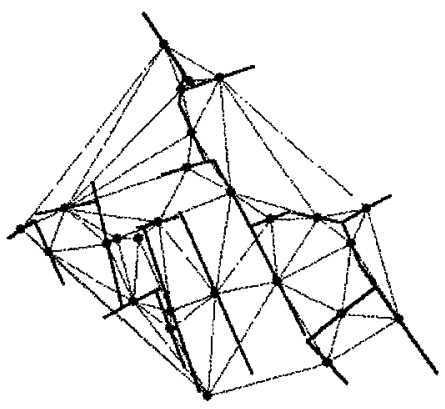

d) .

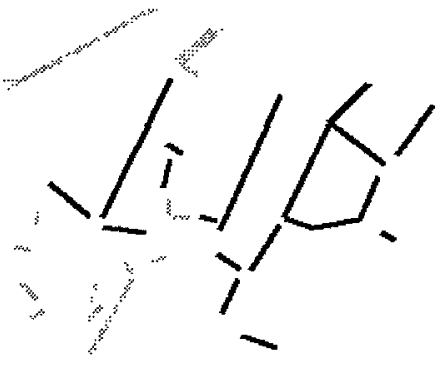

Fig. 1. Integrated grouping-matching process: a) Original image, b) Extracted line segments, c) Initial match, d) Final grouped match.

\section{Conclusions}

We have described a novel approach to relational matching. Rather than performing sequential grouping and matching operations, we accomplish the two processes simultaneously in unified optimisation framework. The dual operations of re-organising the perceptual relation graph and matching it against a model, both optimise a single objective function in the maximum a posteriori probability sense. We have demonstrated the utility of the resulting technique both on the matching of synthetic aperture radar images and on simulated data.

\section{References}

1. K Boyer and A Kak, "Structural Stereopsis for 3D Vision", IEEE PAMI, 10, pp 144-166, 1988.

2. E.R. Hancock and J. Kittler, "Discrete Relaxation," Pattern Recognition, 23, pp.711-733, 1990.

3. R. Horaud and T. Skordas, "Stereo Correspondence through Feature Grouping and Maximal Cliques", IEEE PAMI, 11, pp. 1168-1180, 1989.

4. S. Sarkar and K.L. Boyer, "Perceptual Organisation in Computer Vision: A Review and Proposal for a Classificatory Structure", IEEE SMC, 23, pp 382-399, 1993.

5. R.C. Wilson and E.R Hancock, "Graph Matching by Discrete Relaxation", Pattern Recognition in Practice IV, North Holland pp. 165-177, 1994. 